\title{
Mitochondrial complex activity in permeabilised cells of chronic fatigue syndrome patients using two cell types
}

\author{
Cara Tomas $^{1}$, Audrey Brown ${ }^{1}$, Julia L Newton ${ }^{1,2}$, Joanna L Elson ${ }^{\text {Corresp. } 3,4}$ \\ 1 Institute of Cellular Medicine, Newcastle University, Newcastle upon Tyne, United Kingdom \\ 2 NHS Foundation Trust, Newcastle upon Tyne Hospitals, Newcastle upon Tyne, United Kingdom \\ 3 Institute of Genetic Medicine, Newcastle University, Newcastle upon Tyne, United Kingdom \\ 4 Centre for Human Metabonomics, North-West University, Potchefstroom, South Africa \\ Corresponding Author: Joanna L Elson \\ Email address: j.I.elson@ncl.ac.uk
}

Abnormalities in mitochondrial function have previously been shown in chronic fatigue syndrome (CFS) patients, implying that mitochondrial dysfunction may contribute to the pathogenesis of disease. This study builds on previous work showing that mitochondrial respiratory parameters are impaired in whole cells from CFS patients by investigating the activity of individual mitochondrial respiratory chain complexes. Two different cell types were used in these studies in order to assess individual complex activity locally in the skeletal muscle (myotubes) $(\mathrm{n}=6)$ and systemically (peripheral blood mononuclear cells (PBMCs)) (control $n=6$; CFS $n=13$ ). Complex I, II and IV activity and respiratory activitysupported by fatty acid oxidation and glutaminolysis were measured usingextracellular flux analysis. Cells were permeabilised and combinations of substrates and inhibitors were added throughout the assays to allow states of mitochondrial respiration to be calculated and the activity of specific aspects of respiratory activity to be measured. Results showed there to be no significant differences in individual mitochondrial complex activity or respiratory activity supported by fatty acid oxidation or glutaminolysis between healthy control and CFS cohorts in either skeletal muscle $(p \geq 0.190)$ or PBMCs $(p \geq 0.065)$. This is the first study to use extracellular flux analysisto investigate individual mitochondrial complex activity in permeabilised cells in the context of CFS. The lack of difference in complex activity in CFS PBMCs suggests that the previously observed mitochondrial dysfunction in whole PBMCs is due to causes upstream of the mitochondrial respiratory chain. 


\section{Mitochondrial complex activity in permeabilised cells of 2 chronic fatigue syndrome patients using two cell types}

4 Cara Tomas $^{1}$, Audrey Brown ${ }^{1}$, Julia L. Newton ${ }^{1,2}$, Joanna L. Elson*3,4

$5{ }^{1}$ Institute of Cellular Medicine, Newcastle University, Newcastle upon Tyne, UK

$6 \quad{ }^{2}$ NHS Foundation Trust, Newcastle upon Tyne Hospitals, Newcastle upon Tyne, UK

$7{ }^{3}$ Institute of Genetic Medicine, Newcastle University, Newcastle upon Tyne, UK

$8{ }^{4}$ Centre for Human Metabolomics, North-West University, Potchefstroom, South Africa 


\section{Abstract}

25 Abnormalities in mitochondrial function have previously been shown in chronic fatigue 26 syndrome (CFS) patients, implying that mitochondrial dysfunction may contribute to the 27 pathogenesis of disease. This study builds on previous work showing that mitochondrial 28 respiratory parameters are impaired in whole cells from CFS patients by investigating the 29 activity of individual mitochondrial respiratory chain complexes.

30 Two different cell types were used in these studies in order to assess individual complex activity 31 locally in the skeletal muscle (myotubes) $(n=6)$ and systemically (peripheral blood mononuclear 32 cells (PBMCs)) (control $n=6$; CFS $n=13$ ). Complex I, II and IV activity and respiratory activity 33 supported by fatty acid oxidation and glutaminolysis were measured using extracellular flux 34 analysis. Cells were permeabilised and combinations of substrates and inhibitors were added 35 throughout the assays to allow states of mitochondrial respiration to be calculated and the 36 activity of specific aspects of respiratory activity to be measured.

37 Results showed there to be no significant differences in individual mitochondrial complex 38 activity or respiratory activity supported by fatty acid oxidation or glutaminolysis between healthy control and CFS cohorts in either skeletal muscle $(p \geq 0.190)$ or PBMCs $(p \geq 0.065)$. This is the first study to use extracellular flux analysis to investigate individual mitochondrial complex activity in permeabilised cells in the context of CFS. The lack of difference in complex

42 activity in CFS PBMCs suggests that the previously observed mitochondrial dysfunction in whole PBMCs is due to causes upstream of the mitochondrial respiratory chain. 


\section{Introduction}

49 Chronic fatigue syndrome (CFS), also known as Myalgic Encephalomyelitis (ME), is a debilitating 50 disease affecting $0.2-0.4 \%$ of the population in the UK (1). CFS has a significant impact on the 51 quality of life of patients with key symptoms including severe fatigue and post-exertional 52 malaise $(2,3)$. The mechanisms behind the aetiopathogenesis of CFS are yet to be elucidated.

53 The lack of knowledge of the mechanisms behind the disease contribute to difficulty in 54 obtaining a consensus on diagnostic criteria and the development of widely effective 55 treatments.

56 Various aspects of mitochondrial dysfunction have previously been postulated as contributing 57 to CFS (4-11). Fatigue has been shown to be common in patients with primary mitochondrial 58 disease, (12) however, it should be noted that patients with CFS are not seen to harbour 59 primary mitochondrial mutations (13). Billing-Ross et al. showed that certain changes in the 60 mitochondrial genome increases the likelihood of specific symptoms in CFS patients such as 61 gastrointestinal, neurological, and inflammatory symptoms, however, these genomic changes 62 do not make patients more susceptible to developing the disease (14). Previous studies have 63 shown the energy production, including mitochondrial activity, of whole PBMCs from CFS 64 patients to be significantly impaired compared to a healthy control cohort $(4,15)$. One study, 65 using extracellular flux analysis in whole cells, showed CFS PBMCs to have significantly impaired mitochondrial functioning both under basal conditions and when maximally stimulated to respire, under a number of experimental conditions (4). This suggested that CFS patients were unable to utilise mitochondrial energy production to the same extent as healthy controls and implied that mitochondrial dysfunction may contribute to the pathogenesis of the disease. The study presented here was conducted in order to further investigate if the mitochondrial dysfunction seen in CFS PBMCs was due to atypical activity of individual mitochondrial complexes using the same technique as the previous study (extracellular flux analysis) but in permeabilsied cells rather than whole cells. The use of permeabilised cells allows mitochondria to be directly accessed by the substrates with no cellular interference in terms of substrate transport or intracellular interactions. The permeabilisation of cells also allows the enzymatic activity of individual respiratory chain complexes to be to be measured which cannot be done 
77 easily in whole cells. This study investigates the activity of individual complexes and

78 components of the mitochondrial respiratory chain in myotubes and PBMCs - myotubes were used to investigate mitochondrial activity locally in the skeletal muscle, while PBMCs were used to investigate mitochondrial complex activity systemically. This was achieved by permeabilising cells ensuring that the mitochondria remained intact and using extracellular flux analysis to record oxygen consumption rate of cells following the serial injection of mitochondrial activity altering compounds. Respiratory parameters were calculated and compared between control and CFS derived muscle cells and PBMCs.

For this study, saponin was used to permeabilise the cell membrane. Saponin is a cell permeabilser which acts by forming complexes with cholesterol leading to a reduction in cell membrane integrity, while keeping mitochondrial membranes intact (16). When mitochondria are isolated from cells, the architecture and morphology of the mitochondria is altered (17-20), but permeabilisation of the cell membrane allows the architecture and morphology of mitochondria to remain normal, an advantage over the use of isolated mitochondria as mitochondrial function has previously been shown to have a strong relationship with structure $(21,22)$. Permeabilisation of the cell membrane allows the effect of substrates on mitochondrial activity to be comprehensively assessed by allowing endogenous substrates to be delivered to mitochondria (23). The addition of different substrates and inhibitors alters mitochondrial respiration and allows the activity of individual components of mitochondrial respiration to be measured (24). Originally described by Chance and Williams in 1955, mitochondrial respiratory activity can be measured in terms of several respiratory 'states' (25). State 3 respiration, state 4 respiration and respiratory control ratio (RCR) are often used as markers for mitochondrial respiratory activity. State 3 respiration is when mitochondria have a high concentration of ADP externally, and a high oxygen consumption rate and ATP synthesis thus producing a state whereby ADP stimulated respiration can be measured $(25,26)$. State 4 respiration, on the other hand, is when mitochondria have a very low external ADP concentration, and little or no ATP synthesis due to the complete phosphorylation of ADP to ATP. The respiratory control ratio (RCR) is a measure of the coupling of ATP synthesis and 
105 electron flux and shows the capacity of mitochondria to synthesise ATP via the oxidation of 106 respiratory substrates (27).

107 This work aimed to determine if the enzymatic activity of different complexes of the

108 mitochondrial respiratory chain differed between CFS patients and healthy controls either 109 locally, in the skeletal muscle, or systemically in PBMCs.

111 Materials and methods

\section{Study participants}

113 CFS and control derived primary myoblasts were obtained from muscle biopsies of the vastus

114 lateralis of CFS patients and healthy controls and processed and gifted by Dr Audrey Brown,

115 Newcastle University. All CFS patients fulfilled the Fukuda diagnostic criteria and were recruited

116 via the Newcastle NHS CFS Clinical Service at the Newcastle Hospitals NHS Foundation Trust 117 (28).

118 Blood samples were obtained from patients fulfilling the Fukuda Diagnostic criteria for CFS after 119 obtaining ethical approval from the National Research Ethics Committee North East -

120 Newcastle \& North Tyneside 2 (28). Samples from healthy controls were collected through the

121 Institute of Cellular Medicine (Newcastle University) blood study after obtaining ethical

122 approval from the National Research Ethics Committee North East - County Durham \& Tees

123 Valley. Samples were gathered after informed written consent was obtained.

\section{Reagents}

125 All reagents were obtained from Sigma Aldrich, UK unless otherwise stated.

\section{Cell culture and preparation}

\section{Myotubes}

128 Myoblasts were grown to passage 7 in Ham's F10 medium (Scientific Laboratory Supplies, UK)

129 (supplemented with 20\% fetal bovine serum (FBS) (Life Technologies, UK), 2\% chick embryo 130 extract (Sera Labs International, UK), 2\% penicillin-streptomycin, 1\% amphotericin B). Cells 131 were then seeded at a density of $3 \times 10^{4}$ per well into a 96-well seahorse plate (Agilent 
132 Technologies, UK) in quadruplicate, and differentiated into myotubes in differentiation medium

133 (minimal essential media supplemented with 2\% FBS, 1\% penicillin-streptomycin and 1\%

134 amphotericin B). Experiments were performed after 7 days of differentiation. Differentiation 135 was confirmed by observing the formation of long, multinucleated myotubes in alignment under the microscope.

\section{PBMCs}

138 PBMCs were separated using Histopaque ${ }^{\circledR}$ as described by Tomas et al. (4). The PBMCs used in these experiments were frozen at $-80^{\circ} \mathrm{C}$ in freezing medium $(50 \% \mathrm{RPMI}-1640,40 \% \mathrm{FBS}, 10 \%$ DMSO) and revived and plated the day before experiments. Wells of a 96-well seahorse plate were coated with poly-D-lysine, to aid in the attachment of cells, and left to air-dry for 2 hours

142 prior to the plating of cells. Following revival of cells, PBMCs were seeded at a density of $5 \times 10^{5}$ cells per well in quadruplicate in the poly-D-lysine coated 96-well seahorse plate and incubated overnight in RPMI-1640 (supplemented with 10\%FBS and $1 \%$ penicillin-streptomycin) at $37^{\circ} \mathrm{C}$ and $5 \% \mathrm{CO}_{2}$.

146

147

148

149

150

151

152

153

154

155

156

157

158

159

\section{Extracellular flux analysis}

The XFeg6 analyser (Agilent Technologies) was used to investigate the activity of individual mitochondrial respiratory chain complexes using specific substrates. The protocol used in this study is described by Salabei et al. (24) and the mix, wait and measure times provided by Agilent Technologies (0.5 min/0.5 min/2 min) (29). Seeding densities for PBMCs and myotubes were used as described previously $(4,30)$. Myotubes were seeded at a density of $3 \times 10^{4}$ cells per well while PBMCs were seeded at $5 \times 10^{5}$ per well. On the day of experiments, experimental medium was prepared by supplementing DMEM with $1 \mathrm{mM}$ pyruvate, $2 \mathrm{mM}$ L-glutamine and $1 \mathrm{mM}$ glucose. The $\mathrm{pH}$ of the media was adjusted to 7.4 with $0.1 \mathrm{M} \mathrm{NaOH}$ and warmed to $37^{\circ} \mathrm{C}$. One hour before running the experiment, media was removed from each well of the XFeg6 and replaced with $180 \mu \mathrm{l}$ of prepared medium and incubated for one hour at $37^{\circ} \mathrm{C}$ with no $\mathrm{CO}_{2}$. Mannitol and Sucrose (MAS) buffer (70mM sucrose, $220 \mathrm{mM}$ mannitol, $10 \mathrm{mM}$ potassium phosphate monobasic, $5 \mathrm{mM}$ magnesium chloride, $2 \mathrm{mM}$ HEPES, 1mM EGTA) was prepared. A $4 \mathrm{mg} / \mathrm{ml}$ fatty acid free bovine serum albumin (BSA) solution was created by adding BSA to MAS 
160 to create MAS-BSA buffer. The medium on the plate was replaced with $180 \mu$ I of MAS-BSA 10

161 minutes prior to the plate being loaded into the machine. Oxygen consumption rate (OCR) of

162 cells was measured at 12 points throughout the assay. Three basal readings were made before

163 the first injection containing a mix of the substrate(s) of interest, ADP, FCCP and saponin. Three

164 subsequent readings were made and then the second injection, containing oligomycin, was

165 added to the cells. Another three readings of OCR were made and the final injection of either

166 rotenone or potassium azide was added to the cells, and a final three OCR readings recorded.

167 Saponin concentration was optimised independently for myotubes and PBMCs and the damage

168 to mitochondria caused by saponin was also assessed using cytochrome C (supplementary

169 data). The optimal concentration of saponin for myotubes was determined to be $25 \mu \mathrm{g} / \mathrm{ml}$,

170 while the optimum saponin concentration for PBMCs was $2.5 \mu \mathrm{g} / \mathrm{ml}$. Data were normalised for

171 protein concentration following a bicinchoninic acid (BCA) assay (Fisher Scientific) conducted

172 according to manufacturer's instructions.

173 Compound preparation

174 Compounds and inhibitors used to investigate the activity of different complexes in the

175 mitochondrial respiratory chain are shown in Table 1.

Table 1. Table showing the compounds used to investigate mitochondrial complex activity.

177 Parameter calculations

178 For respiratory chain complex activity, state 3 respiration, state 4 respiration, respiratory

179 control ratio (RCR), basal respiration and maximal respiration were calculated as shown below 180 using the measurement numbers shown in Figure 1.

181

182

Figure 1. Profile of the parameters of mitochondrial respiration measured in isolated mitochondria

183

184 State 3 respiration $=($ average of measurements 4 to 6$)-($ average of measurements 10 to 12$)$

185 State 4 respiration = (average of measurements 7 to 9) - (average of measurements 10 to 12$)$ 
$186 \mathrm{RCR}=\frac{\text { State } 3 \text { respiration }}{\text { state } 4 \text { respiration }}$

187 Data analysis

188 Groups were compared using student's t-tests after confirming equal variances using Levene's 189 test for equality of variances. All statistical tests were carried out using IBM SPSS Statistics 22.

190 Graphs were created using Graphpad Prism 7.

191 Results

192 Myotube respiratory chain complex activity

193 Myotube respiratory chain activity was analysed for state 3 respiration, state 4 respiration, and 194 RCR with the addition of various combinations of substrates and inhibitors. The results for 195 myotubes for complex II respiration (succinate), and complex IV activity (TMPD \& ascorbate) 196 are shown in Figure 2. There were no significant differences between control $(n=6)$ and CFS $197(n=6)$ cohorts for state 3 respiration, state 4 respiration, or RCR when cohorts were compared 198 with student's t-tests $(p \geq 0.190)$.

199

200

Figure 2. State 3 respiration, state 4 respiration, and RCR in control and CFS permeabilised myotubes. Succinate 201 was used to analyse complex II mediated respiration; TMPD \& ascorbate were added to investigate complex IV 202 activity. Groups were compared using student's t-tests. Control $n=6 ;$ CFS $n=6$.

203

204 205

Attempts were made to measure complex I mediated respiration, using pyruvate and malate; respiratory activity supported by fatty acid oxidation, with the addition of palmitoyl-I-carnitine; and respiratory activity supported by glutaminolysis, with the addition of glutamine and malate. Results for all three experiments consistently produced negative values for OCR in both CFS $(n=6)$ and control $(n=6)$ myotubes therefore we were unable to calculate state 3 , state 4 , and RCR for these experiments (Figure 3).

Figure 3. Permeabilised myotube mitochondrial stress test traces. Mitochondrial stress test traces in myotubes permeabilised with the addition of different substrates in the first injection alongside saponin, ADP and FCCP. A. Glutamine and malate. B. Palmitoyl-I-carnitine. C. Pyruvate and malate. Control $n=6 ;$ CFS $n=6$. 
214

215

216

217

218

219

220

221

222

223

224

225

226

227

228

229

230

231

232

233

234

235

236

237

238

239

240

241

\section{PBMC respiratory chain complex activity}

PBMCs from healthy controls and CFS patients were used to investigate different aspects of mitochondrial respiratory chain activity outlined in Table 1. Results showed there to be no difference between state 3 respiration, state 4 respiration or RCR of PBMCs from CFS $(n=13)$ patients and healthy controls ( $n=6$; succinate controls $n=4$ ) for any of the substrate/inhibitor combinations $(p \geq 0.065)$ (

Figure 4).

Figure 4. State 3 respiration, state 4 respiration, and RCR in control and CFS permeabilised PBMCs. Succinate was used to analyse complex II mediated respiration; TMPD \& ascorbate were added to investigate complex IV activity; glutamine \& malate allowed respiratory activity supported by glutaminolysis to be measured; pyruvate \& malate were used to investigate complex I mediated respiration; palmitoyl-I-carnitine was added to assess respiratory activity supported by fatty acid oxidation. Groups were compared using student's t-tests. Control n=6; CFS n=13.

\section{Discussion}

The activity of different aspects of mitochondrial respiratory chain function were investigated by adding various combination of substrates and inhibitors. The effect of the different substrates on state 3 respiration, state 4 respiration and respiratory control ratio (RCR) was measured.

Five different combinations of substrates were investigated for their effects on myotubes and PBMCs - glutamine and malate; palmitoyl-I-carnitine; pyruvate and malate; succinate; TMPD and ascorbate. These were added to investigate respiration supported by glutaminolysis; respiration supported by fatty acid oxidation; complex I activity; complex II activity; and complex IV activity, respectively. Despite there being a lack of difference in OXPHOS between control and CFS cohorts shown in whole myotubes (personal observation GR 2016) (30), these experiments aimed to investigate if more subtle and specific differences occurred in individual complexes. PBMCs were used to see if the specific location of abnormalities identified in whole cells reported previously could be pinpointed to specific complexes or pathways (4). 
242 In myotubes only two of the substrate combinations produced viable results. The addition of

243 pyruvate and malate, palmitoyl-I-carnitine, and glutamine and malate produced negative values

244 for OCR in both CFS ( $n=6)$ and control myotubes $(n=6)$ (Figure 3). The addition of the injected

245 compounds did appear to have an effect on the OCR, but not the anticipated effect, and only

246 achieved the result of producing more negative OCR readings with the addition of each

247 injection and not the expected increase after the first injection. These experiments were

248 repeated a number of times with similar traces produced each time. This suggests that this

249 technique for measuring mitochondrial complex activity in permeabilised cells may not be

250 appropriate for use in myotubes. Successful recordings of the effect of succinate, and TMPD

251 and ascorbate were made in control and CFS myotubes. No difference in state 3 respiration,

252 state 4 respiration, or RCR were seen between the control and CFS cohorts. This indicates that

253 there are no abnormalities in respiratory activity linked to glutaminolysis, or complex IV activity

254 in CFS myotubes. This is in agreement with previous research which, using 3 carboxyl-14C-

255 labelled substrates, found there to be no difference between CFS and control skeletal muscle

256 cell complex I, complex II + III, complex III, or complex IV activity (31). However, given the

257 inconsistency of between substrate readings in this study, with some of the substrates giving

258 successful readings and some not, and the large error bars shown on the traces for each of the

259 substrates (including the substrates for which we could successfully derive state 3 \& 4

260 respiration), the use of this technique to accurately record the activity of specific aspects of

261 mitochondrial respiration in permeabilised myotubes should be questioned. Other techniques

262 such as phosphorescence oxygen sensitive probes and spectrophotometric enzyme assays

263 should be used to analyse the same samples in future experiments in order to determine if

264 these techniques can provide more accurate results than those achieved here with the XFeg6

265 and to see the consistency between techniques. Very few studies have previously been

266 published using extracellular flux analysis to detect mitochondrial activity in permeabilised

267 myotubes. One study used extracellular flux analysis and high resolution respirometry to

268 determine the differences between the techniques (32). The study showed traces from the

269 Seahorse $\mathrm{XF}^{\mathrm{e}} 24$ to have higher levels of variability for each data point which supports the data 
270 reported here showing that extracellular flux analysis may not be the most suitable technique

271 for detecting changes in mitochondrial complex activity in permeabilised myotubes.

272 In PBMCs, successful traces were recorded for all five combinations of substrates. State 3

273 respiration, state 4 respiration, and RCR of control $(n=6$, succinate controls $n=4)$ and CFS $(n=13)$

274 PBMCs were compared. No difference was seen between the two cohorts in any of the

275 substrates investigated. This suggests that the activity of complexes I, II and IV, and respiration

276 supported by fatty acid oxidation or glutaminolysis do not differ in CFS PBMCs compared with

277 healthy controls. These results are in agreement with a study by Lawson et al. who used

278 spectrophotometric techniques to show that permeabilised CFS PBMCs do not differ from

279 healthy controls in terms of complex I, complex II-III, or complex IV activity (8). The consistency

280 of results between the different research groups, using different techniques, strengthens the

281 evidence suggesting that there are no abnormalities in individual mitochondrial complex

282 activity in CFS PBMCs. The lack of differences in PBMCs may suggest that the abnormalities

283 found in whole cells shown previously is not due to abnormalities in the mitochondrial

284

respiratory chain complexes but rather at different points of the respiration pathway such as

285

286

287

288

289

290

291

292

293

294

295

296

297

movement of glucose into cells, AMPK abnormalities, or altered functioning of other

mitochondrial enzymes (4). However, caution must be used in interpreting these results on

their own given the differences in OXPHOS observed in whole PBMCs (4), as the results from

whole cells reflect a more natural and physiologically relevant environment for the

mitochondria. While relatively small sample sizes were used in this study, the consistency of the findings of this study with previous studies using different techniques to measure mitochondrial complex activity in myotubes and PBMCs in CFS patients adds validity to the results $(8,31)$.

\section{Conclusions}

This study investigated the activity of specific components of mitochondrial respiration by looking at individual complex activity and pathways in myotubes and PBMCs in a case-control study. A cell permeabilization protocol developed by Salabei et al. using the XFeg6 extracellular flux analyser was used to conduct these experiments. This is the first study to use extracellular 
298 flux analysis to investigate individual mitochondrial complex activity in permeabilised cells in 299 the context of CFS. Ultimately, normal mitochondrial function was recorded in CFS myotubes 300 and PBMCs, however, relatively small sample sizes were used therefore the results should be 301 interpreted with caution. The finding of normal mitochondrial functioning of CFS myotubes

302 supports the results of unpublished data from whole cells (30). The results showing no

303 difference in mitochondrial activity in permeabilised PBMCs were unexpected given that

304 mitochondrial function in PBMCs has previously been shown to be significantly lower in CFS (4).

305 However, the lack of difference in complex activity in CFS PBMCs is in agreement with results

306 reported by other groups who showed normal mitochondrial respiratory chain complex activity

$307(8,33)$, and postulated that changes in mitochondrial ATP synthesis should be attributed to

308 other causes such as the transport capacity of oxygen (33). Given the results here, the future of

309 bioenergetic studies in CFS should concentrate on mechanisms upstream of the mitochondrial

310 respiratory chain.

311 Acknowledgements

312 The authors would like to thank all the patients and control subjects who participated in this 313 study.

\section{References}

315 1. Chronic fatigue syndrome/myalgic encephalomyelitis (or encephalopathy) - Diagnosis 316 and management of CFS/ME in adults and children, (2007).

317 2. Hvidberg MF, Brinth LS, Olesen AV, Petersen KD, Ehlers L. The health-related quality of

318 life for patients with myalgic encephalomyelitis / chronic fatigue syndrome (ME/CFS). PLoS 319 ONE. 2015;10(7).

320 3. Winger A, Kvarstein G, Wyller VB, Ekstedt M, Sulheim D, Fagermoen E, Småstuen MC,

321 Helseth $\mathrm{S}$. Health related quality of life in adolescents with chronic fatigue syndrome: A cross-

322 sectional study. Health and Quality of Life Outcomes. 2015;13(1).

323 4. Tomas C, Brown A, Strassheim V, Elson JL, Newton J, Manning P. Cellular bioenergetics is 324 impaired in patients with chronic fatigue syndrome. PLOS ONE. 2017;12(10):e0186802.

325 5. Booth NE, Myhill S, McLaren-Howard J. Mitochondrial dysfunction and the 326 pathophysiology of myalgic encephalomyelitis/chronic fatigue syndrome (ME/CFS).

327 International Journal of Clinical and Experimental Medicine. 2012;5(3):208-20.

328 6. Myhill S, Booth NE, McLaren-Howard J. Chronic fatigue syndrome and mitochondrial

329 dysfunction. International Journal of Clinical and Experimental Medicine. 2009;2(1):1-16. 
$3307 . \quad$ Myhill S, Booth NE, McLaren-Howard J. Targeting mitochondrial dysfunction in the 331 treatment of myalgic encephalomyelitis/chronic fatigue syndrome (ME/CFS) - A clinical audit.

332 International Journal of Clinical and Experimental Medicine. 2013;6(1):1-15.

333 8. Lawson NH, CH.; March, D.; Wang, X. Elevated Energy Production in Chronic Fatigue

334 Syndrome Patients. Journal of Nature and Science. 2016;2(10).

335 9. Behan WMH, More IAR, Behan PO. Mitochondrial abnormalities in the postviral fatigue 336 syndrome. Acta Neuropathologica. 1991;83(1):61-5.

337 10. Morris G, Maes M. Mitochondrial dysfunctions in Myalgic Encephalomyelitis/chronic

338 fatigue syndrome explained by activated immuno-inflammatory, oxidative and nitrosative 339 stress pathways. Metabolic Brain Disease. 2014;29(1):19-36.

340 11. Filler K, Lyon D, Bennett J, McCain N, Elswick R, Lukkahatai N, Saligan LN. Association of

341 mitochondrial dysfunction and fatigue: A review of the literature. BBA Clinical. 2014;1:12-23.

342 12. Gorman GS, Elson JL, Newman J, Payne B, McFarland R, Newton JL, Turnbull DM.

343 Perceived fatigue is highly prevalent and debilitating in patients with mitochondrial disease.

344 Neuromuscul Disord. 2015;25(7):563-6.

345 13. Schoeman EM, Van Der Westhuizen FH, Erasmus E, van Dyk E, Knowles CV, Al-Ali S, Ng

346 WF, Taylor RW, Newton JL, Elson JL. Clinically proven mtDNA mutations are not common in 347 those with chronic fatigue syndrome. BMC Med Genet. 2017;18(1):29.

348 14. Billing-Ross P, Germain A, Ye K, Keinan A, Gu Z, Hanson MR. Mitochondrial DNA variants 349 correlate with symptoms in myalgic encephalomyelitis/chronic fatigue syndrome. Journal of 350 Translational Medicine. 2016;14(1).

351 15. Fluge O, Mella O, Bruland O, Risa K, Dyrstad SE, Alme K, Rekeland IG, Sapkota D, Rosland

352 GV, Fossa A, Ktoridou-Valen I, Lunde S, Sorland K, Lien K, Herder I, Thurmer H, Gotaas ME,

353 Baranowska KA, Bohnen LML, Schafer C, McCann A, Sommerfelt K, Helgeland L, Ueland PM,

354 Dahl O, Tronstad KJ. Metabolic profiling indicates impaired pyruvate dehydrogenase function in 355 myalgic encephalopathy/chronic fatigue syndrome. JCl Insight. 2016;1(21).

356 16. Jamur MC, Oliver C. Permeabilization of cell membranes. Methods in Molecular Biology. 357 2010;588:63-6.

358 17. Bach D, Pich S, Soriano FX, Vega N, Baumgartner B, Oriola J, Daugaard JR, Lloberas J, 359 Camps M, Zierath JR, Rabasa-Lhoret R, Wallberg-Henriksson H, Laville M, Palacín M, Vidal H, 360 Rivera F, Brand M, Zorzano A. Mitofusin-2 determines mitochondrial network architecture and 361 mitochondrial metabolism. A novel regulatory mechanism altered in obesity. Journal of 362 Biological Chemistry. 2003;278(19):17190-7.

363 18. Mitra K, Wunder C, Roysam B, Lin G, Lippincott-Schwartz J. A hyperfused mitochondrial 364 state achieved at G1-S regulates cyclin E buildup and entry into S phase. Proceedings of the 365 National Academy of Sciences of the United States of America. 2009;106(29):11960-5.

366 19. Sarin M, Wang Y, Zhang F, Rothermund K, Zhang Y, Lu J, Sims-Lucas S, Beer-Stolz D, Van 367 Houten BE, Vockley J, Goetzman ES, Anthony Graves J, Prochownik EV. Alterations in c-Myc 368 phenotypes resulting from dynamin-related protein 1 (Drp1)-mediated mitochondrial fission.

369 Cell death and disease. 2013;4(6):e670.

370 20. Hagenbuchner J, Kuznetsov AV, Obexer P, Ausserlechner MJ. BIRC5/Survivin enhances

371 aerobic glycolysis and drug resistance by altered regulation of the mitochondrial fusion/fission

372 machinery. Oncogene. 2013;32(40):4748-57. 
373 21. Saks VA, Veksler VI, Kuznetsov AV, Kay L, Sikk P, Tiivel T, Tranqui L, Olivares J, Winkler K, 374 Wiedemann F, Kunz WS. Permeabilized cell and skinned fiber techniques in studies of

375 mitochondrial function in vivo. Molecular and Cellular Biochemistry. 1998;184(1-2):81-100.

376 22. Picard M, Taivassalo T, Ritchie D, Wright KJ, Thomas MM, Romestaing C, Hepple RT.

377 Mitochondrial structure and function are disrupted by standard isolation methods. PLoS ONE.

378 2011;6(3):e18317.

379 23. Clerc $P$, Polster BM. Investigation of mitochondrial dysfunction by sequential

380 microplate-based respiration measurements from intact and permeabilized neurons. PLoS ONE. $3812012 ; 7(4):$ e34465.

382 24. Salabei JK, Gibb AA, Hill BG. Comprehensive measurement of respiratory activity in 383 permeabilized cells using extracellular flux analysis. Nature Protocols. 2014;9(2):421-38.

384 25. Chance B, Williams GR. Respiratory enzymes in oxidative phosphorylation. I. Kinetics of 385 oxygen utilization. Journal of Biological Chemistry. 1955;217(1):383-93.

386 26. Chance B, Williams GR. The respiratory chain and oxidative phosphorylation. Advances 387 in Enzymology and related subjects of biochemistry. 1956;17:65-134.

388 27. Hill BG, Benavides GA, Lancaster JR, Ballinger S, Dell'Italia L, Jianhua Z, Darley-Usmar 389 VM. Integration of cellular bioenergetics with mitochondrial quality control and autophagy. 390 Biological chemistry. 2012;393(12):1485-512.

391 28. Fukuda K, Straus SE, Hickie I, Sharpe MC, Dobbins JG, Komaroff A. The chronic fatigue 392 syndrome: A comprehensive approach to its definition and study. Annals of Internal Medicine. 393 1994;121(12):953-9.

394 29. Agilent Technologies. Assessing Mitochondrial Respiratory Complexes Using Cells 395 Permeabilized with XF Plasma Membrane Permeabilizer (PMP)2016.

396 30. Rutherford G. Investigating the biochemical basis of muscle cell dysfunction in chronic 397 fatigue syndrome: Newcastle University; 2016.

398 31. Smits B, van den Heuvel L, Knoop H, Küsters B, Janssen A, Borm G, Bleijenberg G, 399 Rodenburg R, van Engelen B. Mitochondrial enzymes discriminate between mitochondrial 400 disorders and chronic fatigue syndrome. Mitochondrion. 2011;11(5):735-8.

401 32. Boyle KE, Zheng D, Anderson EJ, Neufer PD, Houmard JA. Mitochondrial Lipid Oxidation 402 is Impaired in Cultured Myotubes from Obese Humans. International Journal of Obesity. 403 2011;36(8).

404 33. Vermeulen RCW, Kurk RM, Visser FC, Sluiter W, Scholte HR. Patients with chronic fatigue 405 syndrome performed worse than controls in a controlled repeated exercise study despite a 406 normal oxidative phosphorylation capacity. Journal of Translational Medicine. 2010;8. 


\section{Table $\mathbf{1}$ (on next page)}

Table showing the compounds used to investigate mitochondrial complex activity 


\begin{tabular}{|c|c|c|c|}
\hline Compound(s) & $\begin{array}{l}\text { 10x port solution } \\
\text { concentration }\end{array}$ & $\begin{array}{l}\text { Final in well } \\
\text { Concentration }\end{array}$ & Complex investigated \\
\hline Pyruvate/malate & $50 \mathrm{mM} / 25 \mathrm{mM}$ & $5 \mathrm{mM} / 2.5 \mathrm{mM}$ & Complex I mediated respiration \\
\hline Succinate & $100 \mathrm{mM}$ & $10 \mathrm{mM}$ & Complex II mediated respiration \\
\hline $\begin{array}{l}\text { Tetamethylphenylendiamine } \\
\text { (TMPD)/Ascorbate }\end{array}$ & $5 \mathrm{mM} / 20 \mathrm{mM}$ & $0.5 \mathrm{mM} / 2 \mathrm{mM}$ & Complex IV activity \\
\hline Palmitoyl-I-carnitine & $0.5 \mathrm{mM}$ & $50 \mu \mathrm{M}$ & $\begin{array}{l}\text { Respiratory activity supported } \\
\text { by fatty acid oxidation }\end{array}$ \\
\hline Glutamine/malate & $40 \mathrm{mM} / 5 \mathrm{mM}$ & $4 \mathrm{mM} / 0.5 \mathrm{mM}$ & $\begin{array}{l}\text { Respiratory activity supported } \\
\text { by glutaminolysis }\end{array}$ \\
\hline ADP & $10 \mathrm{mM}$ & $1 \mathrm{mM}$ & State 3 respiratory activity \\
\hline Saponin & $250 \mu \mathrm{g} / \mathrm{ml}$ & $25 \mu \mathrm{g} / \mathrm{ml}$ & Cell permeabilisation \\
\hline FCCP & $10 \mu \mathrm{M}$ & $1 \mu \mathrm{M}$ & Maximal respiratory activity \\
\hline Oligomycin & $10 \mu \mathrm{M}$ & $1 \mu \mathrm{M}$ & State 4 respiratory activity \\
\hline Rotenone & $10 \mu \mathrm{M}$ & $1 \mu \mathrm{M}$ & Metabolic inhibitor \\
\hline Potassium Azide & $200 \mathrm{mM}$ & $20 \mathrm{mM}$ & Metabolic inhibitor \\
\hline
\end{tabular}


Figure 1

Profile of the parameters of mitochondrial respiration measured in isolated mitochondria

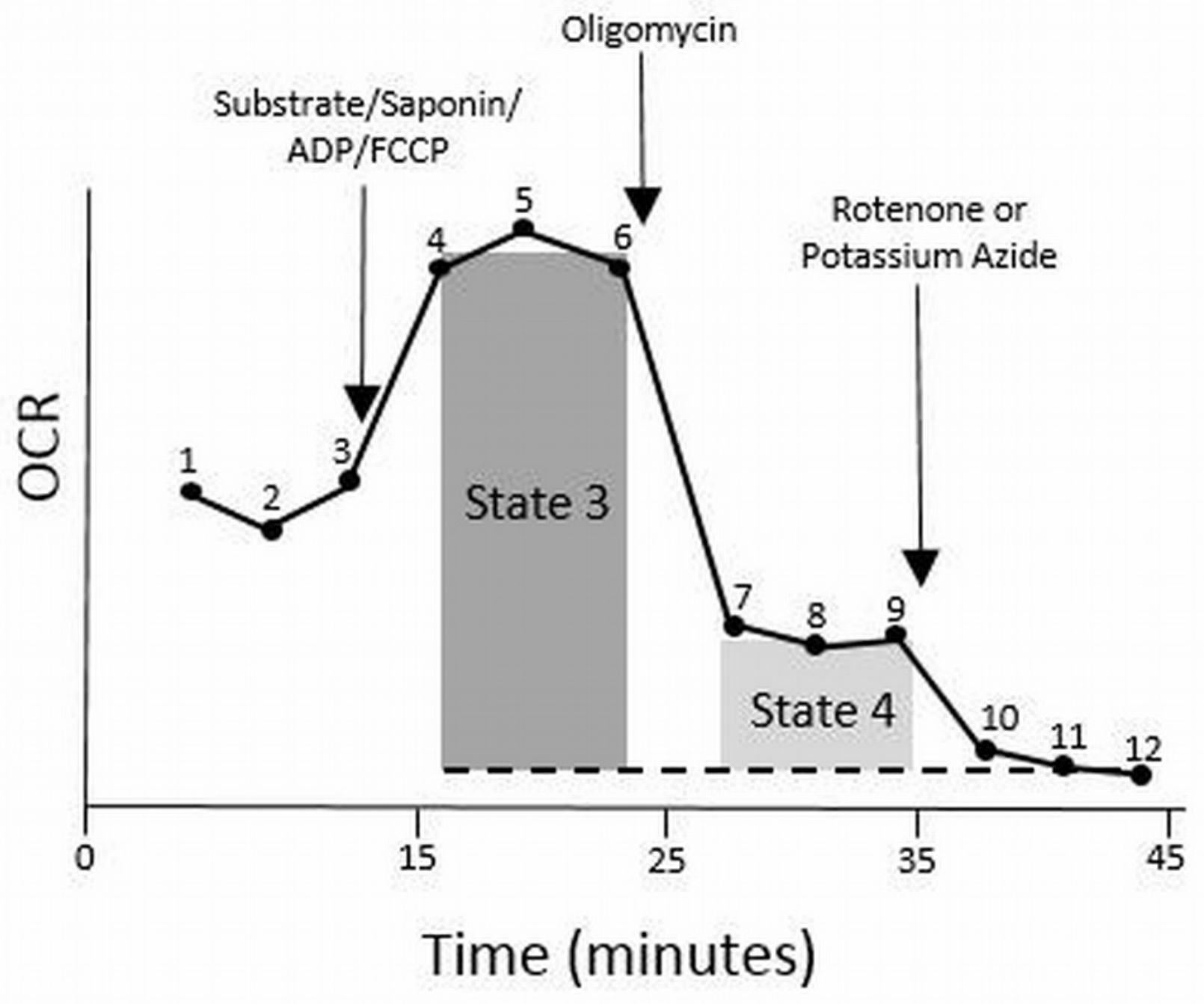


Figure 2

State 3 respiration, state 4 respiration, and RCR in control and CFS permeabilised myotubes

Succinate was used to analyse complex II mediated respiration; TMPD \& ascorbate were added to investigate complex IV activity. Groups were compared using student's t-tests. Control $n=6$; CFS $n=6$.

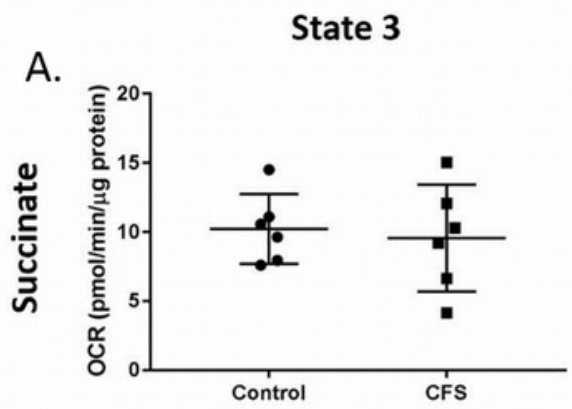

B.

D.

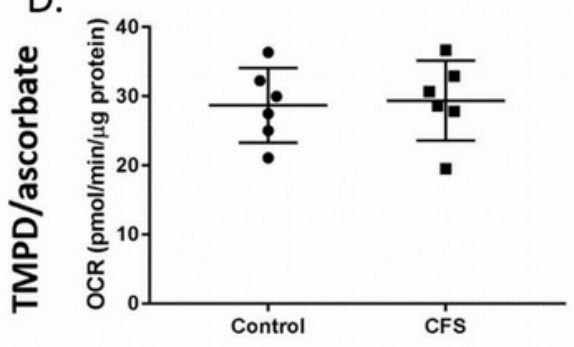

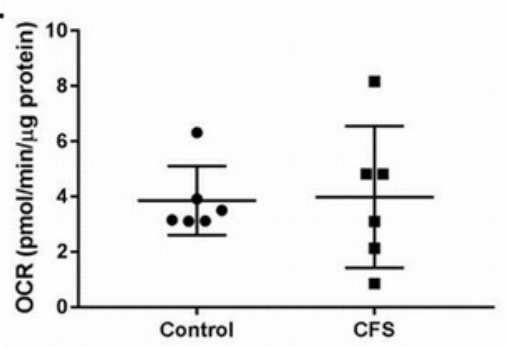

State 4

E.

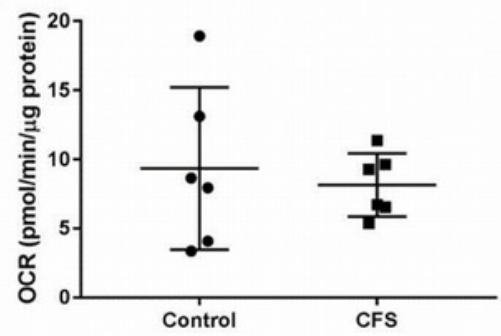

RCR

C.

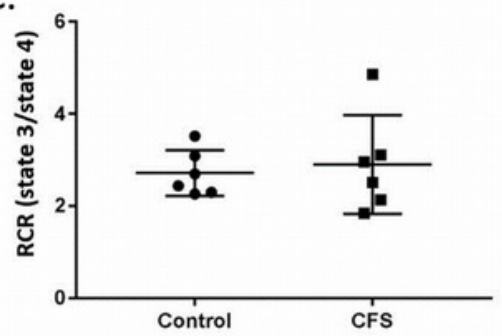

F.

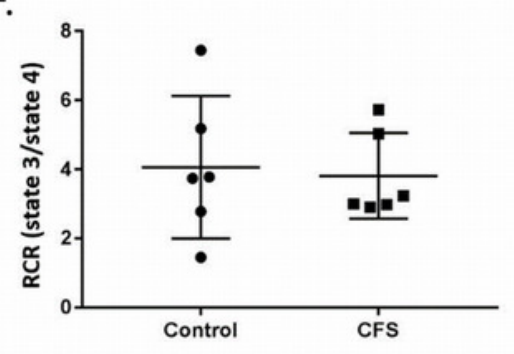


Figure 3

Permeabilised myotube mitochondrial stress test traces

Mitochondrial stress test traces in myotubes permeabilised with the addition of different substrates in the first injection alongside saponin, ADP and FCCP. A. Glutamine and malate.

B. Palmitoyl-I-carnitine. C. Pyruvate and malate. Control $n=6$; CFS $n=6$.

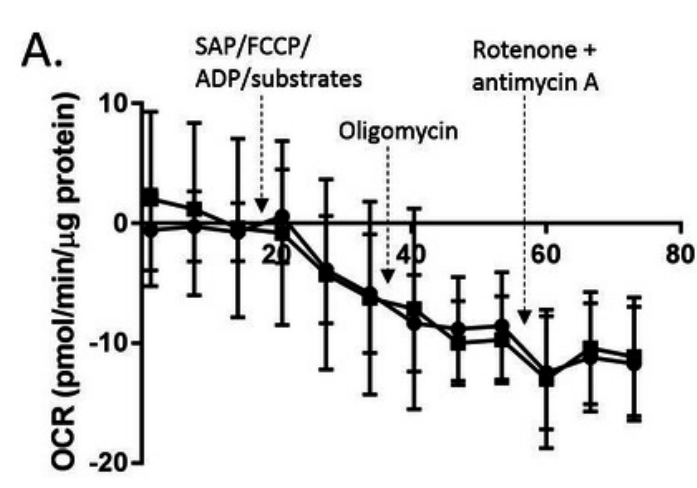

Time (minutes)

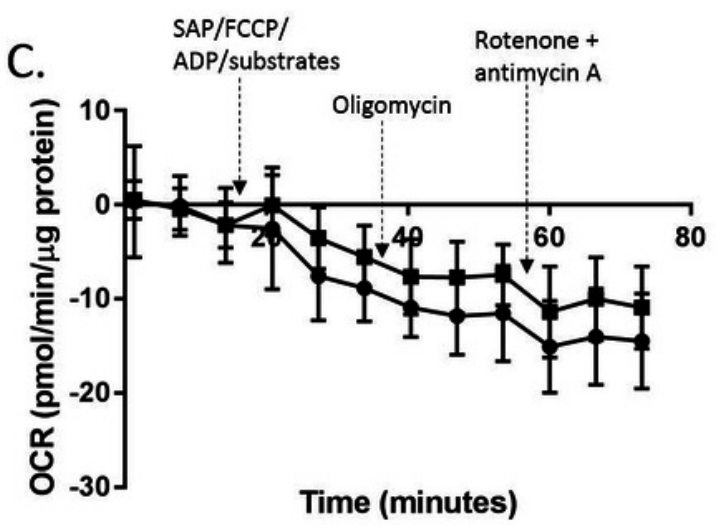

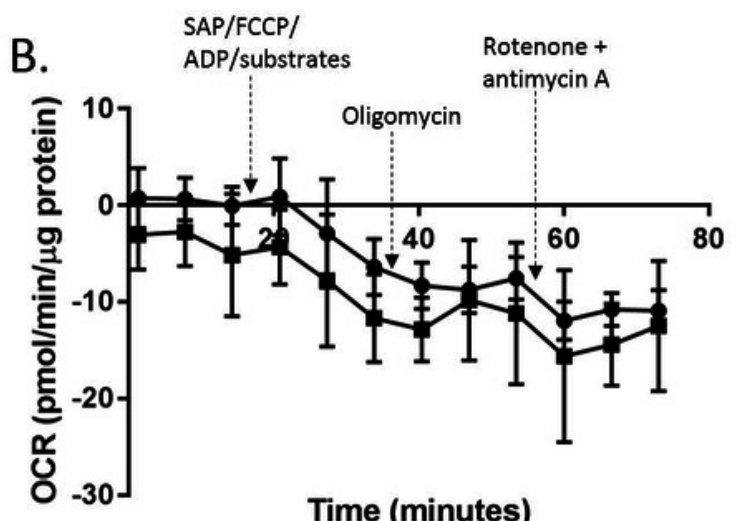

Time (minutes)

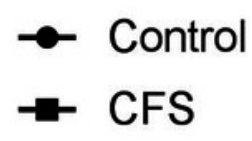




\section{Figure 4}

State 3 respiration, state 4 respiration, and RCR in control and CFS permeabilised PBMCs

Succinate was used to analyse complex II mediated respiration; TMPD \& ascorbate were added to investigate complex IV activity; glutamate \& malate allowed respiratory activity supported by glutaminolysis to be measured; pyruvate $\&$ malate were used to investigate complex I mediated respiration; palmitoyl-I-carnitine was added to assess respiratory activity supported by fatty acid oxidation. Groups were compared using student's t-tests. Control $n=6 ;$ CFS $n=13$. 
State 3

A.

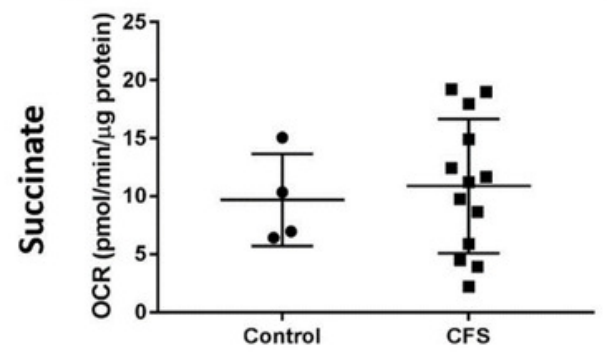

D.

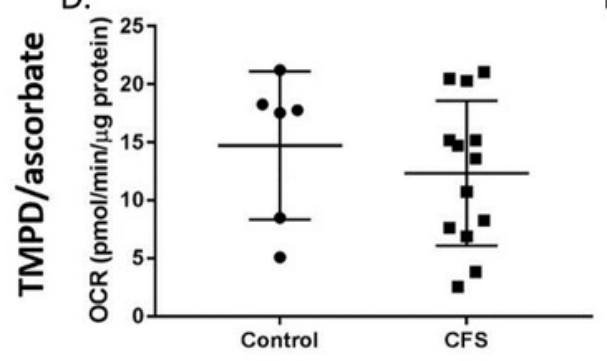

G.

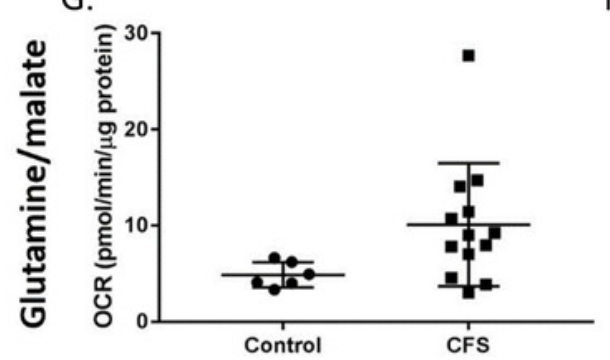

J.

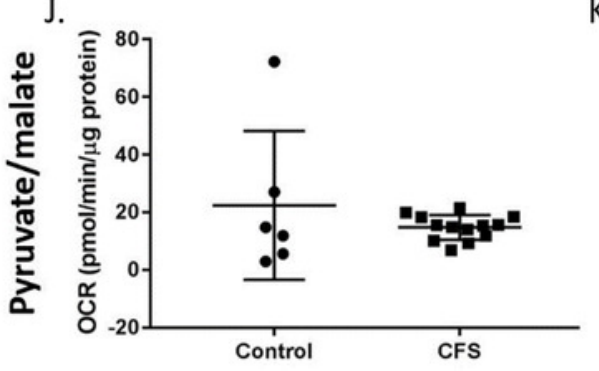

M.

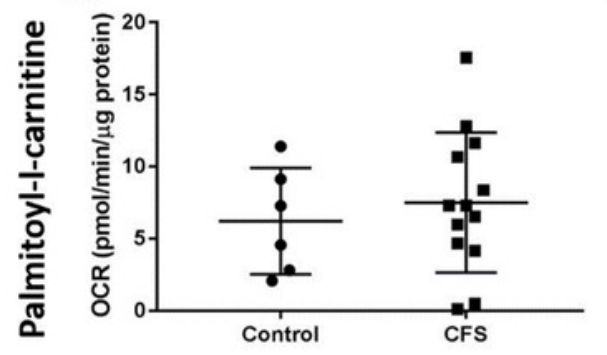

B.

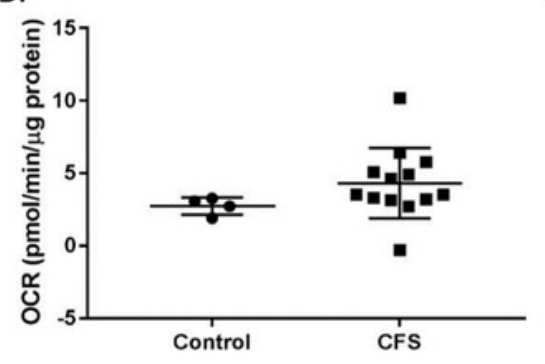

E.

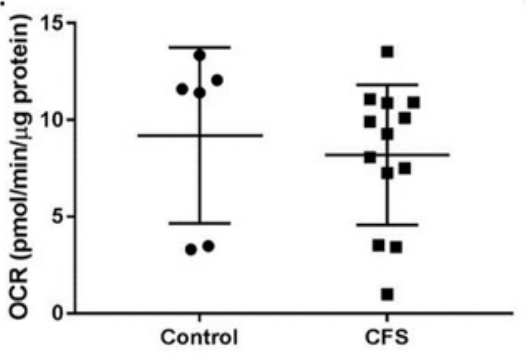

H.

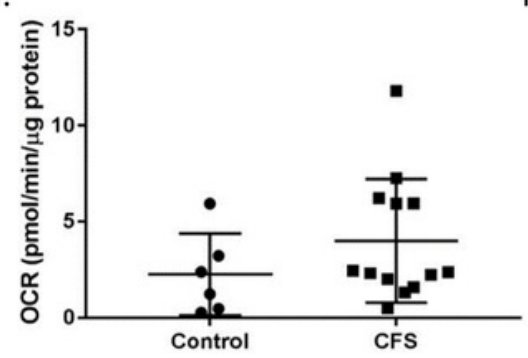

K.

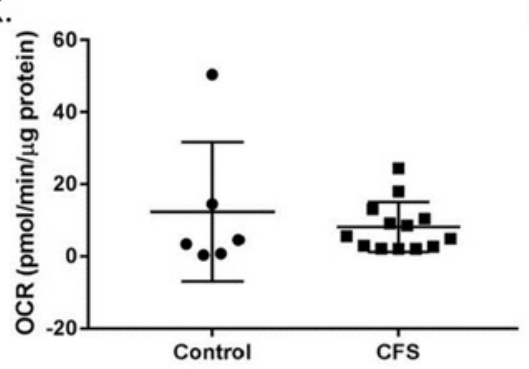

N.

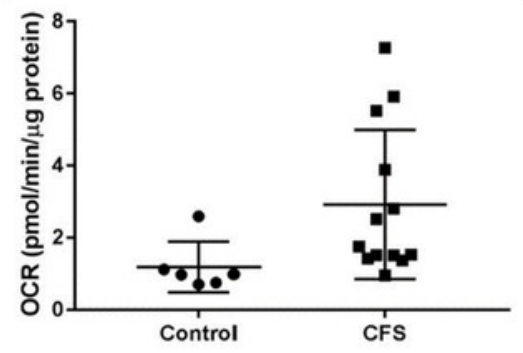

C.

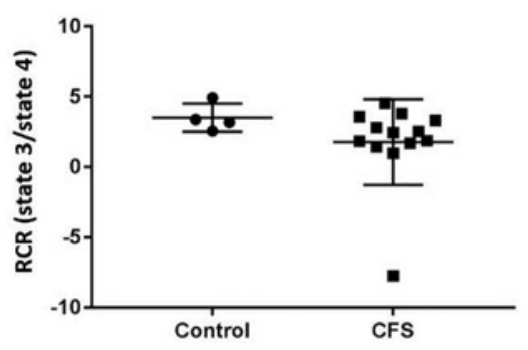

F.

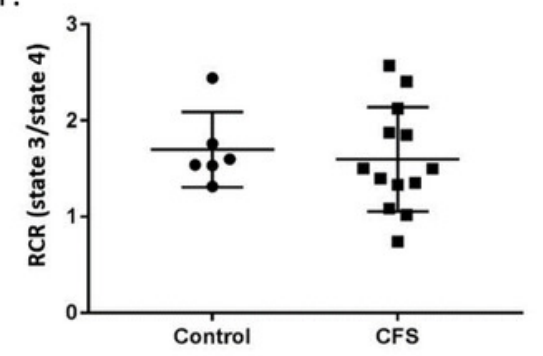

1.

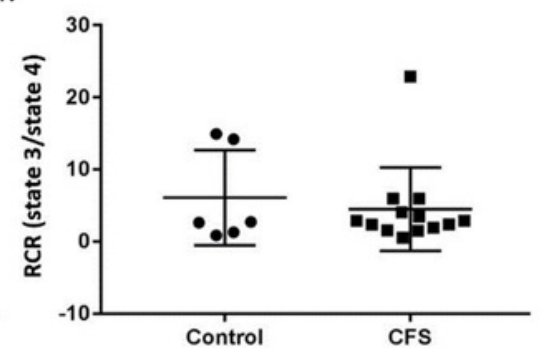

L.

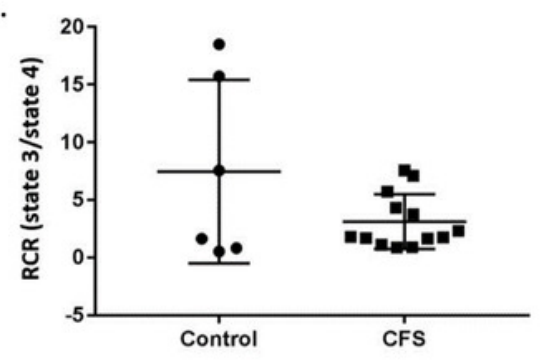

O.

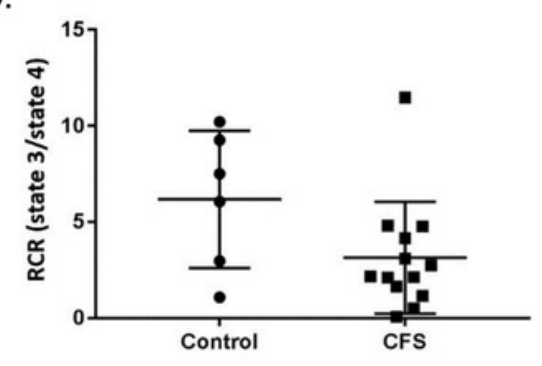

